\title{
Erhöhte Suizidraten bei Psoriasispatienten?
}

\begin{abstract}
Immer wieder wird über die erhöhte Suizidalität von Psoriatikern diskutiert. Eine dänische Studie zeigt jetzt, dass diese Quote differenziert betrachtet werden muss.
\end{abstract}

- ine Psoriasis kann sowohl das Seelen- als auch das Sozialle—ben der Erkrankten schwer belasten und erheblichen Einfluss auf deren Lebensqualität nehmen. Demzufolge geht man von einer erhöhten Suizidalität bei diesen Patienten aus, was einzelne Studien auch nahelegen. Doch in den entsprechenden Untersuchungen wurden vollendete Selbsttötungen, Suizidversuche sowie -gedanken zum Teil gemeinsam erfasst und das Gesamtergebnis einer erhöhten Suizidalität präsentiert. In einer dänischen Kohortenstudie wurde nun der Zusammenhang zwischen Psoriasis und dem Risiko für einen Suizid oder Suizidversuch getrennt untersucht. Hierzu wurden 57.502 Personen mit leichter und 11.009 Personen mit schwerer Psoriasis mit 340.152 gesunden Kontrollprobanden der Allgemeinbevölkerung verglichen.

Von 1997 bis 2011 wurden 280 Fälle von Selbstverletzungen oder Suizidversuchen sowie 574 vollzogene Suizide registriert. In der Rohanalyse mit Patienten aller Schweregrade war das Risiko für Selbstverletzungen oder misslungene Suizidversuche gegenüber den Kontrollprobanden signifikant erhöht (Verhältnis der Inzidenzraten, IRR 1,42), während dies in der adjustierten Analyse nicht mehr signifikant war. Berücksichtigung fanden hier Alter, Geschlecht, sozioökonomischer Status, Alkoholkonsum sowie die Einnahme von Antidepressiva.

Nach den Schweregraden der entzündlichen Hauterkrankung eingeteilt, konnte bei der leichten Form der Psoriasis kein erhöhtes Risiko für Selbstverletzungen oder Suizidversuche festgestellt werden (nicht adjustierte IRR 1,2 bzw. adjustierte IRR 1,01). Bei Patienten mit schwerer Psoriasis dagegen lag die nicht adjustierte IRR bei 2,23 ( $\mathrm{p}=0,002)$ und die adjustierte IRR erreichte 1,69 $(p=0,049)$. Wurde zusätzlich für eine Psoriasis-Arthritis adjustiert, verlor das erhöhte Risiko allerdings seine Signifikanz. Das Risiko für den vollzogenen Suizid war im Vergleich zur Kontrollgruppe weder bei Patienten mit leichter noch mit schwerer Krankheitslast erhöht. Die Selbsttötungen waren von einem Arzt oder durch eine forensische Untersuchung gesichert worden.

Fazit: Psoriatiker haben kein höheres Suizidrisiko als Personen der Allgemeinbevölkerung, und zwar unabhängig von der Schwere ihrer Erkrankung. Es besteht zwar ein gering erhöhtes Risiko für Selbstverletzungen sowie Selbsttötungsversuche, dieses steht aber möglicherweise in Zusammenhang mit dem Vorhandensein einer Psoriasis-Arthritis. Es sei möglich, dass der negative Einfluss der Arthritis auf die Lebensqualität von Psoriatikern zu einer erhöhten Suizidgefahr beiträgt, so die Autoren.

\section{Hier steht eine Anzeige.}

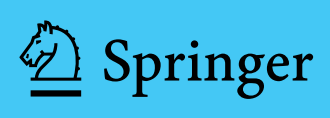

